\title{
Optimal Exit Configuration of Factory Layout for a Safer Emergency Evacuation using Crowd Simulation Model and Multi-Objective Artificial Bee Colony Optimization
}

\author{
Nurulaqilla Khamis ${ }^{1}$, Hazlina Selamat ${ }^{2 *}$, Fatimah Sham Ismail ${ }^{3}$, \\ Omar Farouq Lutfy ${ }^{4}$
}

${ }^{1}$ Malaysia-Japan International Institute of Technology, Universiti Teknologi Malaysia, Jalan Sultan Yahya Petra, 54100, Kuala Lumpur, MALAYSIA

${ }^{2}$ Centre for Artificial Intelligence and Robotics, School of Electrical Engineering, Faculty of Engineering, Universiti Teknologi Malaysia, 81310, Johor Bahru, Johor, MALAYSIA

${ }^{3}$ Control \& Mechatronic Engineering Department, School of Electrical Engineering, Faculty of Engineering, Universiti Teknologi Malaysia, 81310, Johor Bahru, Johor, MALAYSIA

${ }^{4}$ Control and System Engineering Department, University of Technology, Baghdad, IRAQ

*Corresponding Author

DOI: https://doi.org/10.30880/ijie.2019.11.04.020

Received 25 April 2019; Accepted 19 August 2019; Available online 5 September 2019

\begin{abstract}
This work aims at providing a systematic method in producing a safer and optimal factory layout based on a crowd simulation model and the multi-objective artificial bee colony optimization technique. Apart, from ensuring the efficiency of manufacturing processes in planning a factory layout, it is also important that the safety aspect is taken into account. A factory is usually a closed working area consisting of machines, equipment, assembly lines as well as individual working space and other departments within the factory. In this environment, workers move around in the factory to perform different activities, and hence highly complex crowd behaviours that are influenced by the physical, social and psychological factors of the crowd might take place. Therefore, the layout of the factory must be carefully designed so that efficient movements of people can be obtained. Furthermore, during emergency situations that require efficient evacuation of workers from the factory building, a good factory layout will prevent or minimize the possibility of injuries during the evacuation process. This will reduce the evacuation egress time, which is the quantity used to evaluate the evacuation efficiency and the building's level of safety. One of the techniques to assess the evacuation efficiency of a particular space configuration is by using the crowd simulation model. Recent evidences suggest that the representation of crowd dynamics using a simulation model is useful, where experiments with real humans are too dangerous and not practical to be implemented. This work explains the method to provide optimal exit door configurations for a factory layout by analyzing the crowd evacuation time and the discomfort level, where the proposed optimum exit configurations will be compared with the original configuration for a better evacuation efficiency.
\end{abstract}

Keywords: Crowd simulation model, discomfort level, evacuation time, exit configuration, multi-objective optimization 


\section{Introduction}

Crowds occur in open or closed spaces with members of the crowds having preferred or shared common activities [1]. The factory is one of the closed working areas consisting of complex machines, equipment, assembly lines as well as individual working space. In the factory environment, workers move around to accomplish different activities, which may result in highly complex crowd behaviors that are influenced by the physical, social and psychological factors of the crowd. This exposes the workers to various possibilities of overcrowding with serious injuries or even fatalities when they are trapped by accident and become unable to escape in time during emergency evacuation [2]. There are two factors that usually hinder people from evacuating safely, which are the uncontrolled behaviors of the crowd and the inefficient building design [3]. Due to both problems, it will affect evacuation egress time, which is the quantity used to evaluate the evacuation efficiency and the building's level of safety [4]. Since crowd movements and behaviors are very much influenced by their surroundings [5], addressing the issues of a safer building or a space design by providing a good factory layout is vital in order to reduce the number of injuries and loss of lives that might happen for a large crowd during emergency situations. For instance, by placing a suitable location of emergency signage in the environment [6][7], optimal emergency routes [8][9], optimal obstacle placements in large open spaces [10][11] and locations of entrance/exit doors [2][12][13].

One of the possible and appropriate techniques to evaluate safe building designs is by using the crowd evacuation simulation model, which has the capability of including crowd dynamical behaviors and their interactions with the building geometry in the virtual environment. A recent evidence suggests that the representation of crowd dynamics using a simulation model is useful in this study, where experiments with real humans are too dangerous and not practical to be implemented [14]. Moreover, repetitive simulation tests can be done and will be more insightful to help designers with the process of making design suggestions [5].

Crowd evacuation models based on the microscopic approach are commonly used in this research area. The microscopic approach treats each individual in the crowd as an agent, which can be equipped with a certain level of intelligence for decision making. This approach can be implemented using two methods, which are the Cellular Automata (CA) [15][16] and the physical force model [17][18]. In particular, the CA has the limitation of not representing the real behavior of the individual, since the CA model uses the heuristic approach in updating the individual movement rules [19]. One of the physical force models is the Social Force Model (SFM) introduced by Dirk Helbing's [20]. In SFM, the agent motion was governed by Newton's equations. Most recent works have applied the SFM in the simulation analysis as it is capable of simulating the large-medium scale of crowd dynamics including the socio-psychological behaviors under emergency situations.

To provide a safer evacuation plan for a factory layout, exit doors are one of the most crucial design elements that should be optimally designed (in terms of their locations) in a building to improve the building evacuation efficiency. However, most studies focus on the analysis of the exit door position for a single-room environment only [2],[13],[21] and they lack the detailed analysis for multi-room cases such as the complex factory layout. Moreover, a limited systematic optimization approach has been used in finding suitable locations for the exit doors. Most recent works consider the single objective optimization problem in determining the optimal exit door locations, which is minimizing the evacuation time only. It is not comprehensive to use only a single objective optimization to evaluate the evacuation process. Factors of congestion and discomfort definitely will occur and must be considered in the building design stage.

Therefore, the main objective of this paper is to provide a systematic framework for determining the optimal exit door locations for the factory layout in order to achieve a minimum evacuation egress time and discomfort level for the crowd evacuation. The SFM was used as the crowd evacuation model and an optimization algorithm known as the MultiObjective Artificial Bee Colony (MOABC) will be adopted in this work to determine the optimal exit door locations by minimizing two objectives simultaneously.

In the following section, the simulation model used to represent crowd dynamic in the simulated environment is described. This is followed by the description of the optimization of exit door locations using the MOABC algorithm to minimize the evacuation time and the discomfort level. Finally, the results of the optimization process are presented and analyzed.

\section{The Crowd Evacuation Model based on the Social Force Model}

In SFM, they represent the movement of individual in the crowd using Newton's equations of motions as described in Equation (1) to (2).

$$
\begin{gathered}
\sum F=m_{i} a \\
m_{i} a=f^{0}+f_{i j}+f_{i w}
\end{gathered}
$$


The individual movement will be influenced by three types of forces which are the motivational force to move $\left(\mathrm{f}^{0}\right)$, the repulsion force among other individuals $\left(\mathrm{f}_{\mathrm{ij}}\right)$ and the repulsion force among obstacles $\left(\mathrm{f}_{\mathrm{iw}}\right)$. For $\mathrm{f}^{0}$, the movement of each individual $i$ with a mass $m_{i}$ will be influenced with a direction, $e^{0}(t)$, actual speed, $v_{i}(t)$, and the desired speed, $v_{i}^{0}(t)$, in respective time, $\tau_{i}$. By using Equation (3), the rate of change of the individual's speed and the summation of three forces can be calculated as follows:

$$
m_{i} \frac{d v_{i}}{d t}=m_{i} \frac{v_{i}^{0}(t) e_{i}^{0}-v_{i}(t)}{\tau_{i}}+\sum_{j(\neq i)} f_{i j}+\sum_{w} f_{i w}
$$

To represent the socio-psychological behaviour of the body compression and the sliding friction force in individual movements, the interaction can be included as shown in Equation (4).

$$
\begin{gathered}
\mathrm{f}_{\mathrm{ij}}=\mathrm{A}_{\mathrm{i}} \mathrm{e}^{\frac{\left(\mathrm{r}_{\mathrm{i}}-\mathrm{d}_{\mathrm{ij}}\right)}{\mathrm{B}_{\mathrm{i}}}} \mathrm{n}_{\mathrm{ij}}+ \\
\operatorname{kg}\left(\mathrm{r}_{\mathrm{ij}}-\mathrm{d}_{\mathrm{ij}}\right) \mathrm{n}_{\mathrm{ij}}+\mathrm{kg}\left(\mathrm{r}_{\mathrm{ij}}-\mathrm{d}_{\mathrm{ij}}\right) \Delta \mathrm{v}_{\mathrm{ji}}^{\mathrm{t}} \mathrm{t}_{\mathrm{ij}}
\end{gathered}
$$

In Equation (4), $A_{i} e^{\frac{\left(r_{i}-d_{i j}\right)}{B_{i}}} n_{i j}$ represents the repulsion force with other individuals, where $A_{i}$ and $B_{i}$ are constants, while $d_{i j}=\left\|x_{i}-x_{j}\right\|$ denotes the center of body masses of individual $i$ and $j$. The summation of body radius $r_{i j}$ for individuals $i$ and $j$ can be represented by $r_{i j}=r_{i}+r_{j}$. The $n_{i j}=\left(n_{i j}^{1}, n_{i j}^{2}\right)=\frac{x_{i}-x_{j}}{\left\|x_{i}-x_{j}\right\|}$ is the normalized vector pointing from individual $\mathrm{j}$ to $\mathrm{i}$. To address the movement of crowd during emergency situation, the individual body compression $\mathrm{k}\left(\mathrm{r}_{\mathrm{ij}}-\mathrm{d}_{\mathrm{ij}}\right) \mathrm{n}_{\mathrm{ij}}$ and the sliding friction force $\mathrm{k}\left(\mathrm{r}_{\mathrm{ij}}-\mathrm{d}_{\mathrm{ij}}\right)$ have been introduced in the mathematical model where, $\mathrm{k}$ and $\mathrm{k}$ are constants, $\Delta v_{j i}^{t}=\left(v_{j}-v_{i}\right) t_{i j}$ is the tangential velocity difference and $t_{i j}=\left(-n_{i j}^{2}, n_{i j}^{1}\right)$ represents the tangential direction. The function of $g(x)$ is equal to zero if the individuals do not touch each other, where $\left(r_{i j}<d_{i j}\right)$. All the constant values used in the SFM model are given in Table 1. These values were based on the validated real experiments on evacuation scenes from previous literature [22]. The body radii of the individuals in the simulations have random values between $0.25 \mathrm{~m}$ to $0.35 \mathrm{~m}$, which are based on the values used in previous literature [20]. The desired speed for each individual to escape is set to $v_{i}^{0}=1.5 \mathrm{~ms}^{-1}$, as found in [20].

\section{Table 1 - Parameters used in the Social Force Model}

\begin{tabular}{cc}
\hline Parameters & Values \\
\hline $\mathrm{A}_{\mathrm{i}}$ & $998.97 \mathrm{~N}$ \\
$\mathrm{~B}_{\mathrm{i}}$ & $0.08 \mathrm{~m}$ \\
$\tau$ & $0.5 \mathrm{~s}$ \\
$\mathrm{k}$ & $819.62 \mathrm{~kg} / \mathrm{s}^{2}$ \\
$\mathrm{k}$ & $510.49 \mathrm{~kg} / \mathrm{ms}$ \\
$\mathrm{r}$ & $0.25 \mathrm{~m}-0.35 \mathrm{~m}$ \\
$\mathrm{v}_{\mathrm{i}}^{0}$ & $1.5 \mathrm{~ms}^{-1}$ \\
\hline
\end{tabular}

In addition, Equation (5) represents the interaction forces between the individual and the wall or the obstacle in the simulated environment. The notation of $\mathrm{d}_{\mathrm{iw}}$ represents the distance between the individual $\mathrm{i}$ and the wall/obstacle, $\mathrm{n}_{\mathrm{iw}}$ and $\mathrm{t}_{\mathrm{iw}}$ denote the perpendicular direction and the tangential direction to the wall, respectively.

$$
\begin{aligned}
f_{i w}= & A_{i} e^{\frac{\left(r_{i}-d_{i w}\right)}{B_{i}}} n_{i w}+k g\left(r_{i}-d_{i w}\right) n_{i w} \\
& +k g\left(r_{i}-d_{i w}\right)\left(-v_{i} t_{i w}\right) t_{i w}
\end{aligned}
$$

From the simulation model, the level of discomfort can be quantified by using Equation (6), where D is varies from $0 \leq \mathrm{D} \leq 1$. In particular, the D value reflects the frequency and the degree of sudden velocity changes, i.e the level of discontinuity of walking due to necessary avoidance maneuvers.

$$
\mathrm{D}=\frac{1}{\mathrm{~N}} \sum_{\mathrm{i}} \frac{\overline{\left(\mathrm{v}_{1}-{\overline{\mathrm{v}_{1}}}^{2}\right.}}{\overline{\left(\mathrm{v}_{1}^{2}\right)}}=\frac{1}{\mathrm{~N}} \sum_{\mathrm{i}}\left(1-\frac{\overline{\mathrm{v}}^{2}}{\left({\overline{\mathrm{v}_{1}}}^{2}\right)}\right)
$$




\section{Multi-Objective Artificial Bee Colony Optimization (MOABC)}

The optimization problem is very common in many engineering fields. This problem might possess more than one objective function, known as the multi-objective problem, which will be involved in this work. Optimization is a technique to select the best solutions among candidates according to the criteria being tested [23]. Swarm intelligence is one of the most common approaches that has been applied in the optimization problems. There are several algorithms based on the swarm intelligence approach such as Particle Swarm Optimization (PSO), Ant Colony Optimization (ACO), Artificial Bee Colony (ABC) algorithms, etc. These algorithms have been widely used to solve multi-objective problems in different areas. The Multi-Objective Artificial Bee Colony (MOABC) algorithm is employed in this work due to its simplicity and fast converging using fewer control parameters compared to other techniques [24]. It is used to optimize the locations of exit doors for the factory layout considered in this work so as to achieve minimum evacuation time to evacuate and discomfort level.

Basically, the structure of MOABC algorithm is based on the foraging behaviour of honey bees [24]. It employs a population of different types of bees in a D-dimensional search space to find the optimum solution. The employed bee task is to bring nectar information of the explored food source back to the hive. Next, the onlooker bee in the hive will choose a good food source by watching the "waggle dance" from the employed bee. Finally, the scout bee will fly spontaneously to search a new food source. The food source, $\mathrm{i}$ position vector $\mathrm{x}_{\mathrm{i}}=\left(\mathrm{x}_{\mathrm{i} 1}, \mathrm{x}_{\mathrm{i} 2}, \ldots, \mathrm{x}_{\mathrm{iD}}\right)$ represents a possible solution to the related problem meanwhile the nectar information is a cost function in the algorithm. The cost function is used to evaluate the quality (fitness) of the associated solutions. The aim of most cases in multi-objective optimization problems is to search for the maximum or the minimum value of the cost function as the target solution. Since there are more than one objective function involved, multi-objective problems become harder to solve. In these problems, there is no unique solution, instead a set of acceptable trade-off optimum solutions is produced by using the pareto-front. There is no unique solution instead of producing a set of acceptable trade-off optimum solutions by using pareto-front. One objective is sacrificed if an improvement on the other objective is to be made [23]. This process will be repeated iteratively until there is no better solution available. Therefore, a curve of pareto optimal front is produced and this value will be chosen as the final result of the optimization.

\section{Implementation of Multi-Objective Artificial Bee Colony Optimization in the Problem}

In this section, the MOABC algorithm is employed to optimize exit door locations for the factory layout so as to achieve minimum evacuation time to evacuate and discomfort level. The multi-objective with a one-dimensional problem (to determine positions of exit doors) is considered. The sizes of the doors are maintained at $1 \mathrm{~m}$ throughout the simulation and optimization evaluation. The fitness value or the quality (evacuation time and discomfort level) of the solutions (exit door locations) is evaluated by using the SFM. A detailed flowchart for the optimization process is illustrated in Fig. 1.

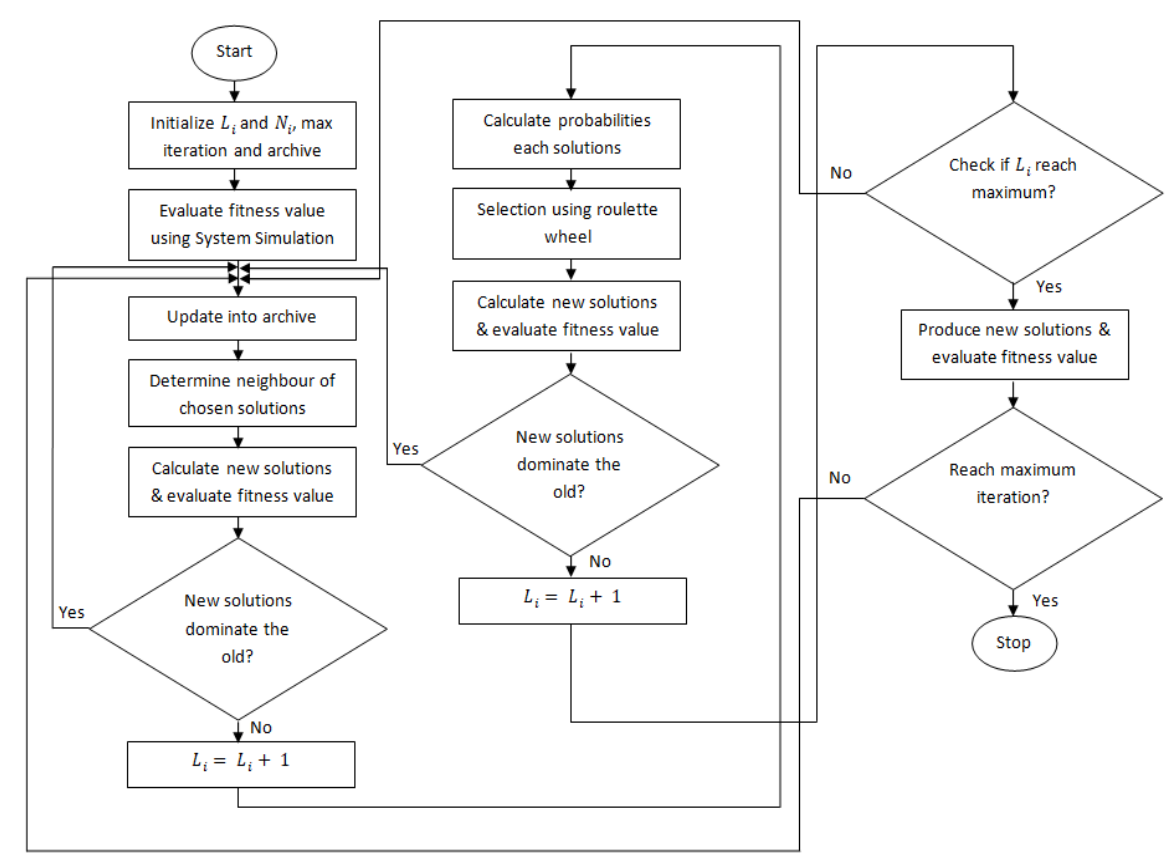

Fig. 1 - Flowchart of MOABC optimization algorithm 
This algorithm consists of 5 phases, which include the initialization phase, the employed bee phase, the onlooker bee phase, the scout bee phase and the archive update.

\subsection{Initialization Phase}

Firstly, define the values of control parameters involved in the algorithm, which are the number of population (exit door locations), the limit, number of iterations and the fixed archive size. The exit door locations are randomly initialized by using Equation (7). Where $\mathrm{i}$ is equal to one as our problem is one-dimensional problem and $\mathrm{n}$ solution, $\mathrm{n}=$ $1, \ldots, \mathrm{SN}\left(\mathrm{SN}=\right.$ number of population). The value of $\mathrm{x}_{\mathrm{n}, \mathrm{i}}$ is bounded between specified lower and upper values, specifically $\mathrm{l}_{\mathrm{i}}=0$ and $\mathrm{u}_{\mathrm{i}}=$ room length respectively.

$$
\mathrm{x}_{\mathrm{n}, \mathrm{i}}=\mathrm{l}_{\mathrm{i}}+\operatorname{rand}(0,1) *\left(\mathrm{u}_{\mathrm{i}}-\mathrm{l}_{\mathrm{i}}\right)
$$

Next, the solutions retrieved from $x_{n, i}$ will be evaluated using the SFM Equation (1) - (6) and the objective function values of the evacuation time and the discomfort level are calculated. The best solutions will be stored inside a fixed archive size.

\subsection{Employed Bee Phase}

In this phase, each employed bee phase will use the best archive solutions and create its own solution. This calculation can be done by using Equation (8).

$$
v_{n, i}=x_{n, i}+\emptyset_{n, i}\left(x_{n, i}-x_{m, i}\right)
$$

Where $\mathrm{m} \in(1, \ldots, \mathrm{SN}), \mathrm{m} \neq \mathrm{n}$ and $\mathrm{i} \in(1, \ldots, \mathrm{D})$ are randomly chosen indexes. $\emptyset_{\mathrm{n}, \mathrm{i}}$ is a random number within the range $[-1,1]$. By producing new exit door locations from $v_{n}$, the objective function is calculated using the SFM Equation (1) - (6). Next, by using non-dominated sorting algorithm, if the new solution is better than the corresponding existing one in the archive, the solutions will be replaced. Otherwise, the limit value will be increased by one.

\subsection{Onlooker Bee Phase}

After optimizing the archive solutions by the employed bees, they will share their information with onlooker bees. The onlooker bee needs to choose the solutions from the employed bee by calculating the probability $P_{k}$ using the following equation:

$$
\mathrm{P}_{\mathrm{k}}=\frac{\text { fit }\left(\mathrm{x}_{\mathrm{m}}\right)}{\sum_{\mathrm{m}=1}^{\text {Solution number }} \text { fit }\left(\mathrm{x}_{\mathrm{k}}\right)}
$$

Where, fit $\left(\mathrm{x}_{\mathrm{k}}\right)$ is the probability of the solution proposed by the employed bee $\mathrm{m}$ that is proportional to the quality of the solution. fit $\left(\mathrm{x}_{\mathrm{k}}\right)$ can be formulated using Equation (10).

$$
\text { fit }\left(\mathrm{x}_{\mathrm{k}}\right)=\frac{\operatorname{dom}(\mathrm{k})}{\text { Solution Number }}
$$

Where, $\operatorname{dom}(\mathrm{k})$ is the function that returns the number of solutions dominated by solution $\mathrm{k}$. Next, the onlooker bee will choose the probability by using the roulette wheel selection and randomly choose the candidate in the archive. Finally, a new position will be calculated using Equation (8), and then this position objective function will be obtained using the SFM Equations from (1) to (6). If the solution dominates the old value, the solution will be updated in the archive.

\subsection{Scout Bee Phase}

The scout bee will determine the abandoned solution by checking the limit parameter that was previously defined in the initialization phase. If the maximum trial limit for a given bee is reached, that bee becomes a scout bee that will find a new exit door position using Equation (7), evaluate the objective function and check whether the solution dominates the archive value or not. If this condition is satisfied, the solution will be replaced, otherwise the old value remains in the archive. 


\subsection{Archive Update}

Archive is the place where all the best solutions will be compared and stored by using the non-dominated algorithm as shown in Equation (11) - (14). At each iteration and through each phase involved in MOABC, every new solution will be compared with other solutions to omit any dominated solution so only the best solution (non-dominated) is maintained in the archive. From here, pareto-front can be plotted where it shows the best solutions inside the archive.

$$
\begin{gathered}
\text { Evacuation time }: \min f_{i}(x) \\
\text { Discomfort Level }: \min f_{j}(x) \\
x^{*} \in \mathbb{S} \\
i \neq j \\
f_{i}(x)=f_{i}\left(x^{*}\right), f_{j}(x)<f_{j}\left(x^{*}\right)
\end{gathered}
$$

Where;

$$
\begin{array}{rll}
x & = & \text { Decision variable position vector } \\
x^{*} & = & \text { Non-dominated solution of a multi-objective optimization problem } \\
\mathbb{S} & = & \text { Feasible region in the search space }
\end{array}
$$

\section{Simulation Results and Discussion}

\subsection{Simulation Data}

Fig. 2 represents the factory layout that is used as our experiment test for the proposed method. The factory layout size is set to $30 \mathrm{~m} \times 15 \mathrm{~m}$ and for the number of individuals in the factory, we take maximum of 60 people that can occupy the space. The locations of the individuals will be randomly distributed in the environment. The main exit door of the factory plan will be on the left, while the doors of other rooms will be at the center. The pathway size is set to $5 \mathrm{~m}$ and the exit door size is set to $1 \mathrm{~m}$. Fig. 3 illustrates the locations of the exit doors implemented in the simulation model.

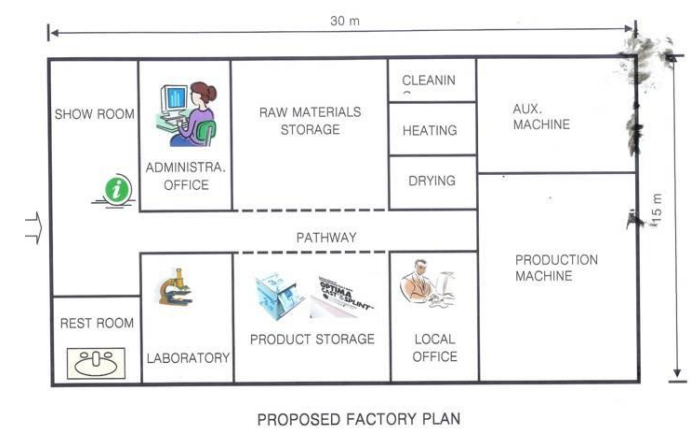

Fig. 2 - Original factory layout

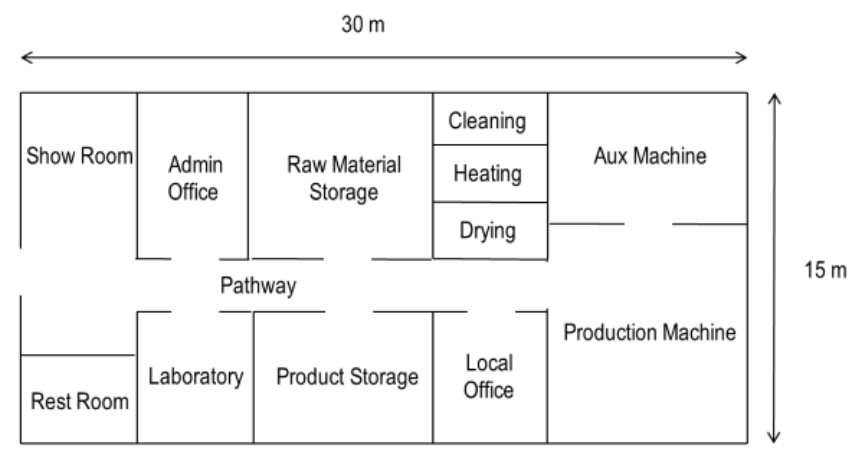

Fig. 3 - Original factory layout exit door locations 
Meanwhile for optimization parameters, the number of population, limit, maximum iterations and archive is set to $10,50,100$ and 30 , respectively.

\subsection{Results and Analysis}

The objective function in multi-objective problem is the evacuation time and discomfort level and it is evaluated by using the SFM. The simulation was processed by using a Personal Computer (PC) with Intel® Core TM i7-4770 CPU. Fig. 4 illustrates the pareto-front for the best solutions stored in the archive. The user can freely choose from the pareto set whose results will satisfy the needs. Table 2 below shows the comparison of multi-objective solutions for the evacuation time and the discomfort level from the original design and those proposed by the optimizer. Fig. 5 illustrates the comparison arrangement of the exit door for original layout and proposed by the optimizer.

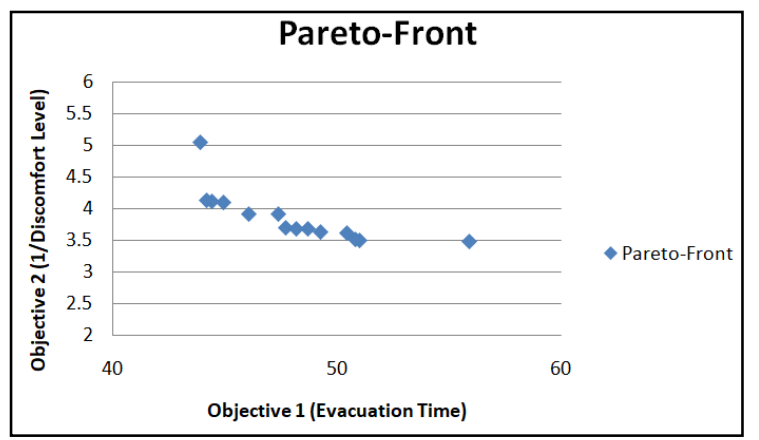

Fig. 4 - Pareto-front for evacuation time and discomfort level optimization

Table 2 - Comparison between the locations of the original layout and those proposed by the optimizer

\begin{tabular}{lccc}
\hline & $\begin{array}{c}\text { Door } \\
\text { Location }\end{array}$ & $\begin{array}{c}\text { Evacuation } \\
\text { Time }\end{array}$ & Discomfort \\
\hline $\begin{array}{l}\text { Original } \\
\begin{array}{l}\text { Layout } \\
\text { Proposed by } \\
\text { the optimizer } \\
\text { (pareto-front) }\end{array}\end{array}$ & Center & $51.45 \mathrm{~s}$ & 0.243 \\
\hline
\end{tabular}

(a)

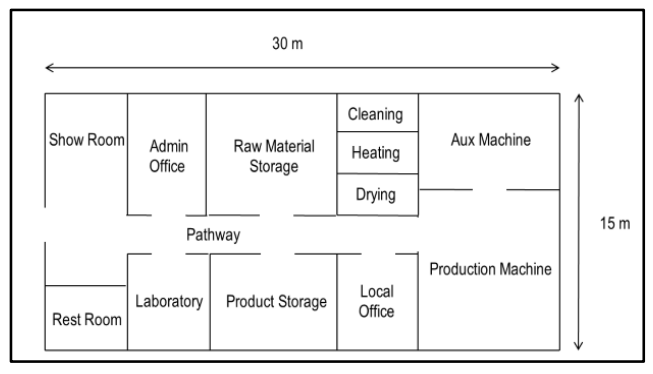

(b)

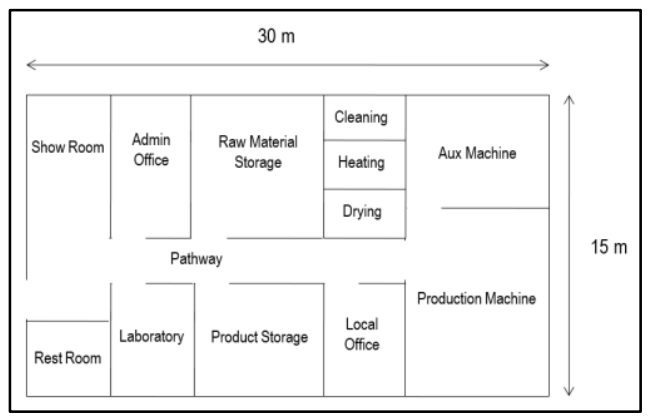

Fig. 5 - (a) Exit door arrangement for original layout; (b) Exit door arrangement proposed by the optimizer 
In order to analyze and compute the performance for the proposed exit door locations by the multi-objective optimizer and the original exit door locations, detailed analysis on the number of people left at different evacuation time is depicted in Fig. 6. The result clearly shows that proposed exit door locations using the optimizer gave an improvement in evacuation time. People leave more quickly by using the proposed solutions from the pareto set due to lower body compression to escape in front of the main exit door. If higher body compression occurs in front of the main exit door, it will cause higher discomfort level and hence a delay in time for the crowd to evacuate.

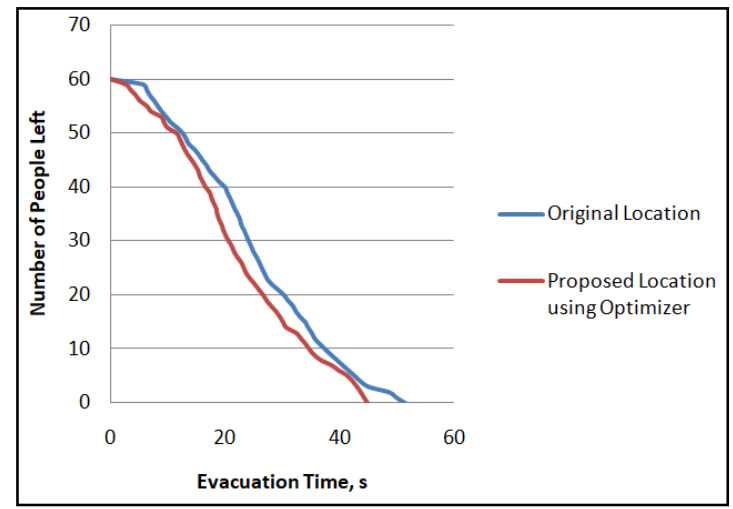

Fig. 6 - Comparison number of people left vs. evacuation time between the original and the proposed exit door locations using the optimizer

\section{Conclusion and Future Work}

The main motivation behind this study was to find an optimum location for the exit door in a factory layout for a better evacuation efficiency. The multi-objective problem whose task is to minimize the evacuation time and the discomfort level was proposed in this work. More precisely, the Multi-Objective Artificial Bee Colony (MOABC) optimization and the Social Force Model (SFM) were employed in this study to find the most suitable solutions. A set of pareto-front was used to archive the best solutions and the user can freely choose the most preferable solution. It is clearly demonstrated that the proposed exit door location by using the multi-objective optimizer can speed up the evacuation time and minimize the discomfort level. As a result, it is proven that the proposed method can improve the evacuation efficiency. More complex environments including different structures of obstacles could be implemented in the future.

\section{Acknowledgement}

The authors would like to thank Universiti Teknologi Malaysia and the Ministry of Higher Education for their supports. This project is supported by Research University Grant Vote Q.K130000.2509.13H78.

\section{References}

[1] N. Khamis, H. Selamat, R. Yusof, and F. S. Ismail, "Magnetic force model approach with path finding feature for an improved crowd movement simulation," in Communications in Computer and Information Science, 2017.

[2] J. Wu, X. Wang, J. Chen, G. Shu, and Y. Li, "The position of a door can significantly impact on pedestrians' evacuation time in an emergency," Appl. Math. Comput., vol. 258, pp. 29-35, 2015.

[3] S. Gwynne, E. R. Galea, M. Owen, P. J. Lawrence, and L. Filippidis, "A review of the methodologies used in the computer simulation of evacuation from the built environment," Build. Environ., vol. 34, no. 6, pp. 741-749, 1999.

[4] E. D. Kuligowski, R. D. Peacock, and B. L. Hoskins, "Technical Note 1680 A Review of Building Evacuation Models , 2nd Edition," Secretary, 2010.

[5] Cabinet Office, Understanding Crowd Behaviours, vol. 59. 2009.

[6] M. L. Chu, P. Parigi, J. C. Latombe, and K. H. Law, "Simulating effects of signage, groups, and crowds on emergent evacuation patterns," AI Soc., vol. 30, no. 4, pp. 493-507, 2015.

[7] K. Yenumula, C. Kolmer, J. Pan, and X. Su, "BIM-Controlled Signage System for Building Evacuation," Procedia Eng., vol. 118, pp. 284-289, 2015.

[8] Q. Li, Z. Fang, Q. Li, and X. Zong, "Multiobjective evacuation route assignment model based on genetic algorithm," in Geoinformatics, 2010 18th International Conference on, 2010, pp. 1-5.

[9] L. D. Han, F. Yuan, S. M. Chin, and H. Hwang, "Global optimization of emergency evacuation assignments," Interfaces (Providence)., vol. 36, no. 6, pp. 502-513, 2006.

[10] E. Cristiani and D. Peri, "Handling obstacles in pedestrian simulations: Models and optimization," 2015. 
[11] B. Piccoli and A. Tosin, "Pedestrian flows in bounded domains with obstacles," Contin. Mech. Thermodyn., vol. 21, no. 2, pp. 85-107, 2009.

[12] P. Kamkarian and H. Hexmoor, "Exploiting the imperialist competition algorithm to determine exit door efficacy for public buildings," Simulation, 2013.

[13] T. Huan-Huan, D. Li-Yun, and X. Yu, "Influence of the exits' configuration on evacuation process in a room without obstacle," Phys. A Stat. Mech. its Appl., vol. 420, pp. 164-178, 2015.

[14] N. W. F. Bode and E. A. Codling, "Human exit route choice in virtual crowd evacuations," Anim. Behav., vol. 86, no. 2, pp. 347-358, 2013.

[15] X. Zheng, W. Li, and C. Guan, "Simulation of evacuation processes in a square with a partition wall using a cellular automaton model for pedestrian dynamics,” Phys. A Stat. Mech. its Appl., vol. 389, no. 11, pp. 2177-2188, 2010.

[16] W. Yuan and K. H. Tan, "Cellular automata model for simulation of effect of guiders and visibility range," Curr. Appl. Phys., vol. 9, no. 5, pp. 1014-1023, 2009.

[17] X. Yang, H. Dong, Q. Wang, Y. Chen, and X. Hu, "Guided crowd dynamics via modified social force model," Phys. A Stat. Mech. its Appl., vol. 411, pp. 63-73, 2014.

[18] M. Moussa??d, N. Perozo, S. Garnier, D. Helbing, and G. Theraulaz, "The walking behaviour of pedestrian social groups and its impact on crowd dynamics," PLoS One, vol. 5, no. 4, 2010.

[19] K. Teknomo, "Microscopic Pedestrian Flow Characteristics: Development of an Image Processing Data Collection and Simulation Model," 2002

[20] D. Helbing, I. Farkas, and T. Vicsek, "Simulating dynamical features of escape panic," Nature, vol. 407, no. 6803, pp. 487-490, 2000.

[21] Z. Nuo, J. Bin, S. Chun-Fu, and Y. Hao, "Simulation of pedestrian evacuation based on an improved dynamic parameter model," Chinese Phys. B, vol. 21, no. 5, p. 50501, 2012.

[22] M. Li, Y. Zhao, L. He, W. Chen, and X. Xu, "The parameter calibration and optimization of social force model for the real-life 2013 Ya'an earthquake evacuation in China," Saf. Sci., vol. 79, pp. 243-253, 2015.

[23] A. F. A. Rahman, H. Selamat, F. S. Ismail, and N. Khamis, "Power consumption optimization of a building using multiobjective particle swarm optimization,” J. Teknol., vol. 72, no. 2, 2015.

[24] D. Karaboga, B. Gorkemli, C. Ozturk, and N. Karaboga, “A comprehensive survey: Artificial bee colony (ABC) algorithm and applications," Artif. Intell. Rev., vol. 42, no. 1, pp. 21-57, 2014. 